\title{
Long-term sensitization training primes Aplysia for further learning
}

\author{
Evangelos G. Antzoulatos, Marcy L. Wainwright, Leonard J. Cleary, and John H. Byrne ${ }^{1}$ \\ Department of Neurobiology and Anatomy, W.M. Keck Center for the Neurobiology of Learning and Memory, The University of \\ Texas Medical School at Houston, Texas 77030
}

\begin{abstract}
Repetitive, unilateral stimulation of Aplysia induces long-term sensitization (LTS) of ipsilaterally elicited siphon-withdrawal responses. Whereas some morphological effects of training appear only on ipsilateral sensory neurons, others appear bilaterally. We tested the possibility that contralateral morphological modifications may have functional significance. Therefore, we examined whether LTS training primes subsequent sensitization. Twenty-four hours after LTS training the effects of brief shock treatment (BST) were examined. BST failed to sensitize animals that had previously received either 4-d control treatment or 4-d ipsilateral LTS training. In contrast, BST did sensitize animals that had previously received 4-d contralateral LTS training, suggesting the presence of a latent trace that primes the animal for further learning.
\end{abstract}

The siphon withdrawal reflex of Aplysia is a useful model system for understanding the neurobiology of learning and memory. The reflex exhibits sensitization, a simple form of learning, in which responses elicited by weak test stimuli are augmented by training with strong, usually noxious, stimuli (Carew et al. 1971; Pinsker et al. 1973; Scholz and Byrne 1987). Short-term sensitization (lasting from seconds to minutes) relies on covalent modification of pre-existing proteins. Intermediate-term sensitization (from minutes to hours) requires translation of mRNA into protein but does not require gene transcription. Long-term sensitization (LTS) (lasting days) requires both gene transcription and translation (Sutton et al. 2001). A critical locus of learning-related plasticity is the glutamatergic synapse of sensory neurons onto target motor neurons (Antzoulatos and Byrne 2004). Several experimental observations have suggested that LTS is partly mediated by structural changes of sensory neurons, which maintain sensorimotor synapses in a facilitated state (Bailey and Chen 1983; Wainwright et al. 2002).

Twenty-four hours after LTS training on the lateral body wall of intact Aplysia, the siphon withdrawal reflex is sensitized only when evoked by test stimuli on the side of the tail ipsilateral to the trained body wall. When test stimulation is delivered to the contralateral side of the tail, siphon responses are normal (i.e., non-sensitized), similar to the responses of untrained animals (Wainwright et al. 2002). The ipsilateral effect of unilateral training suggests that a critical part of memory may be stored in the ipsilateral pleural-pedal ganglia, that is, the part of the Aplysia central nervous system that mediates the afferent limb of the reflex. Consequently, sensory neurons of only the ipsilateral ganglia would be expected to change after LTS training. Indeed, the number of sensorimotor appositions and the amplitude of sensorimotor excitatory postsynaptic potentials increased only on the ipsilateral side of trained animals. Surprisingly, other structural features of sensory neurons, such as the number of varicosities and the length of neurites, did change on the contralateral side (Wainwright et al. 2002, 2004). The functional importance of these changes was not apparent, however. One possibility is that the dormant structural elements may set the stage for sub-

1Corresponding author.

E-mail John.H.Byrne@uth.tmc.edu; fax (713) 500-0621.

Article published online before print. Article and publication date are at http:// www.learnmem.org/cgi/doi/10.1101/lm.230306. sequent learning. This study tested the hypothesis that unilateral LTS training primes Aplysia for further sensitization.

The animal training and testing procedures have been described previously in detail (Wainwright et al. 2002). Briefly, the tail-elicited siphon withdrawal reflex was evoked by weak electrical stimulation of the tail. Test stimuli $(20 \mathrm{~ms} \mathrm{AC})$ were delivered bilaterally through Teflon-coated silver wires implanted $1 \mathrm{~cm}$ rostral to the tip of the tail and $0.5 \mathrm{~cm}$ lateral to the midline (Fig. 1A). The intensity of test stimuli was adjusted to $2 \times$ reflex threshold. Training started after the recording of baseline behavior (five test stimuli on each side at 10-min intervals, alternating on the two sides of the tail). A single training session consisted of four trains $(1 \mathrm{~Hz}, 10 \mathrm{sec})$ of sensitizing stimuli $(500 \mathrm{~ms}, 60 \mathrm{~mA}$ AC) repeated at 30-min intervals (Fig. 1B). Sensitizing stimuli were delivered to the lateral body wall outside the receptive field of tail sensory neurons. Four identical training sessions were performed at 24-h intervals. The post-training behavioral measurement was performed $24 \mathrm{~h}$ after the last day of training, on day 5, and was identical to the pre-training testing session. After each test stimulus, the duration of siphon withdrawal, from onset of contraction to onset of relaxation, was measured by a "blind" observer, using a stopwatch. Averages of the five test scores before and after LTS training were calculated, and their ratio was used for betweengroup comparisons of the training effect (post/pre ratio). All experiments were conducted at $15^{\circ} \mathrm{C}$. Data were analyzed with oneway ANOVA, followed by Student-Newman-Keuls post hoc comparisons. Values are reported as means \pm SEM.

Four daily sessions of sensitization training led to a robust increase in the duration of siphon withdrawal elicited by weak stimulation of the ipsilateral side of the tail (Fig. 2). The average post/pre ratio of response duration was significantly larger for test stimuli delivered to the ipsilateral side of trained animals than for those delivered to the contralateral side of trained animals or to untrained animals (Ipsi, $2.61 \pm 0.38, n=22$; Contra, $1.38 \pm 0.15, n=20$; Untrained, $1.35 \pm 0.18, n=21$; $F_{(2,60)}=7.64, P<0.005$; individual post hoc comparisons: Ipsi vs. Contra, $q=4.68, P<0.05$; Ipsi vs. Untrained, $q=4.84, P<0.05$; Contra vs. Untrained, $q=0.10, P=0.95)$. The data indicate that, as also observed previously (Wainwright et al. 2002, 2004), LTS training produced a lateralized enhancement of the withdrawal response.

Two minutes after the last test stimulus, a subset of animals received brief strong stimulation (two 500-ms pulses, $1 \mathrm{~Hz}, 60$ 
A.

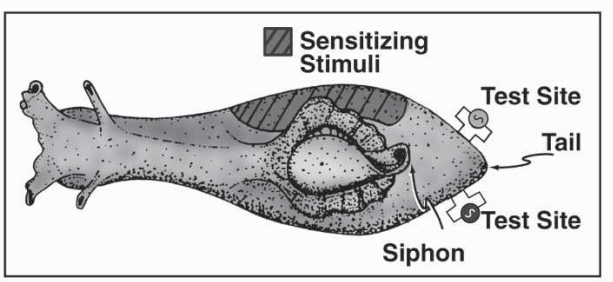

B.

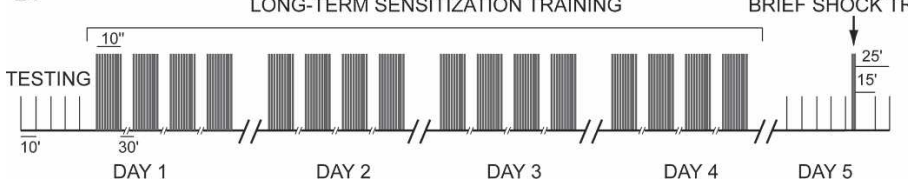

Figure 1. Protocol for sensitization training. (A) All training stimuli were delivered on a randomly chosen side of the lateral body wall. Testing stimuli were delivered on the ipsilateral and contralateral sides of the tail, at an area more caudal to the area (lateral body wall) to which training stimuli were delivered. (B) Four-day training for LTS started immediately after a session of baseline behavioral testing. Twenty-four hours after the end of training, an identical behavioral testing session was used to assess the extent of LTS. Brief shock treatment (BST) was conducted after the end of the post-LTS testing session, either on the side that had received LTS training (Ipsi), or on the opposite side of LTS training (Contra). BST training was also conducted on animals that did not receive LTS training, but had previously been tested in two sessions $5 \mathrm{~d}$ apart (Untrained). The effects of BST were assessed 15 and 25 min post-treatment.

mA AC) to the lateral body wall (Fig. 1B), outside the receptive field of tail sensory neurons. This brief shock treatment (BST) was selected to induce minimal sensitization, if any, and therefore accentuate between-group differences in learning. Because the side to receive BST was randomly selected, some animals that had been previously trained for LTS received BST on the same side (Ipsi), whereas other animals that had been previously trained for LTS received BST on the opposite side (Contra). The "Untrained" group of animals had not received any LTS training prior to BST. Sensitization was assessed with additional test stimulation on the side receiving BST, 15 and 25 min after BST (Fig. 1). The effects of BST were expressed as the ratio of each response (15 and $25 \mathrm{~min}$ after training) to the average response duration of the five tests delivered to that side prior to BST (i.e., the post-LTS behavioral measurements).

Fifteen minutes after BST the average post/pre ratio was not significantly different among the three groups of animals (Fig. 3; Ipsi, $1.41 \pm 0.52, n=5$; Contra, $1.56 \pm 0.55, n=9$; Untrained, $\left.1.11 \pm 0.40, n=8 ; F_{(2,19)}=0.22, P=0.80\right)$. In contrast, $25 \mathrm{~min}$ after BST the average post/pre ratio of the Contra group was significantly larger than that of the other two groups (Ipsi, $0.95 \pm 0.22, n=6$; Contra, $2.14 \pm 0.39, n=9$; Untrained, $1.00 \pm 0.11, n=8 ; F_{(2,20)}=5.70, P<0.05 ;$ individual post hoc comparisons: Ipsi vs. Contra, $q=3.97, P<0.05$; Ipsi vs. Untrained, $q=0.18, P=0.89$; Contra vs. Untrained, $q=4.11$, $P<0.05)$.

The absence of sensitization 25 min after BST in the Ipsi group (Fig. 3) may have been due to a ceiling effect. LTS of siphon withdrawals, induced by 4 -d ipsilateral training, may have prevented any further augmentation of the siphon withdrawal reflex. Indeed, the responses of the Contra group of animals $25 \mathrm{~min}$ after BST did not differ significantly from those of the Ipsi group (Ipsi, 9.29 $s \pm 2.69, n=6$; Contra, $10.99 s \pm 1.95, n=9$; $\left.t_{(13)}=0.53, P=0.61\right)$. These data collectively suggest, therefore, that 4-d LTS training primes the contralateral side to display a form of sensitization, the expression of which is delayed more than $15 \mathrm{~min}$. The lack of this priming effect on the ipsilateral side may be due to saturation.
A dominant view in the field of learning and memory, dating back to Ramón y Cajal, is that long-term memory relies, at least in part, on structural modifications of neuronal terminals and formation of new synapses (Lamprecht and LeDoux 2004). This doctrine was most clearly supported by experimental data in the 1980s, when the number and size of sensory neuron synapses were found to change after long-term sensitization in Aplysia (Bailey and Chen 1983). Although the association of structural modifications with long-term plasticity or memory has been repeatedly replicated (Bailey and Chen 1988a,b, 1989; Wainwright et al. 2002, 2004; Kim et al. 2003), not all training protocols that produce LTS also produce neurite outgrowth (Wainwright et al. 2002). Moreover, training can also lead to axonal restructuring without apparent learning (Wainwright et al. 2002, 2004). If these structural changes do not mediate overt memory, they may have latent effects on learning.

Although latent learning has been studied extensively in mammals, primarily in the context of maze solving and classical conditioning (Tolman 1949; Lubow and Moore 1959), it has not been adequately addressed in invertebrates. If present in simple model systems, like Aplysia, latent learning could be readily analyzed at the cellular and molecular levels. An instance of latent learning in Aplysia was recently reported by Philips et al. (2006), who found that animals that had been previously trained for LTS displayed facilitated acquisition of further learning after the original long-term memory had decayed with time (i.e., memory savings). Moreover, animals that had failed to show long-term memory following training, subsequently displayed facilitated acquisition of sensitization. It is noteworthy that in the Philips et al. (2006) study, LTS was induced with a single training session,

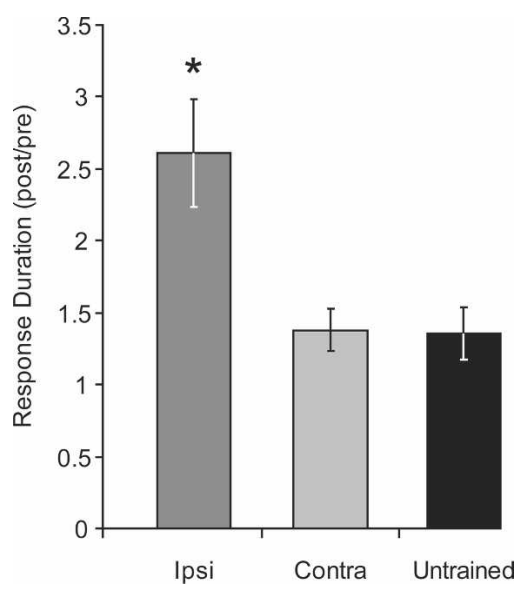

Figure 2. Long-term sensitization of tail-elicited siphon withdrawal reflex. Sensitization of the tail-elicited siphon withdrawal reflex is apparent $24 \mathrm{~h}$ after 4-d LTS training, only when the ipsilateral side (Ipsi) is tested. When the contralateral side (Contra) is tested, the reflex is similar to that elicited by stimulation of untrained animals. Long-term memory for sensitization of the tail-elicited siphon withdrawal reflex, therefore, is only expressed unilaterally. ${ }^{*} P<0.05$. 


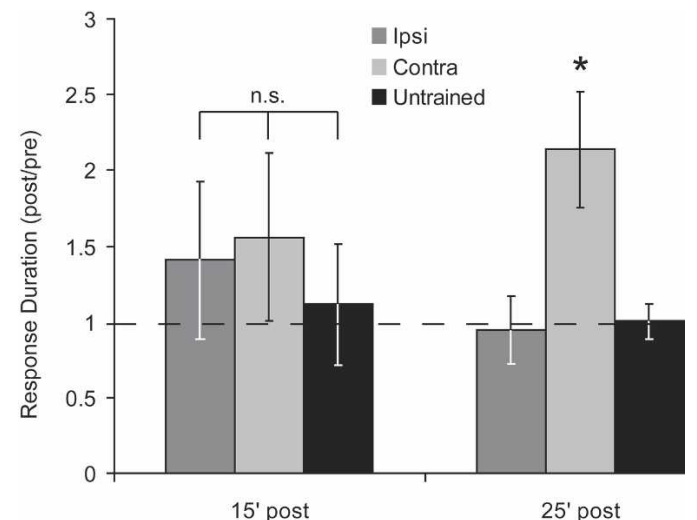

Figure 3. Long-term sensitization training primes the animal for further learning. One day after the end of 4-d LTS training, subsequent brief shock treatment (BST) had variable effects, depending on the prior training history and time after testing. Fifteen minutes after BST, there was no difference among the animals that had previously received ipsilateral LTS (dark grey), contralateral LTS (light grey), or no LTS (black). In contrast 25 min after BST, a significant difference emerges among the three groups of animals. The BST had a significantly larger effect on the animals that had previously received LTS training on the contralateral side. Despite the lack of overt memory, shown in Figure 2, 4-d LTS training does affect the animal's contralateral side in a latent way, manifested as a priming of further sensitization. ${ }^{*} P<0.05$.

which does not induce detectable morphological modifications in sensory neurons (Wainwright et al. 2002).

The conclusion of our study is consistent with the previously reported findings of Philips et al. (2006). Specifically, LTS training of Aplysia has not only overt but also latent learning effects. The results reported here indicate that a brief training stimulus, which was not sufficient to induce sensitization, can do so if it is preceded by 4-d training on the opposite side of the animal's body. The critical comparison in this study is between the animals that received no LTS training (Untrained group) and the ones that received 4-d LTS on the contralateral side (Contra group). Even though in neither of the two groups did LTS training have apparent learning effects (Fig. 2), the additional treatment on day 5 revealed that the two groups were not equivalent (Fig. 3). Instead of inducing overt long-term memory, 4-d contralateral training had a priming effect, manifested in the animal's potential to display further sensitization. The reason there was no apparent priming of sensitization on the ipsilateral side is not clear, but it may be due to a ceiling effect of prior LTS to limit either the induction or expression of additional sensitization.

It is not clear whether the sensitization that appeared 25 min after BST is short-term or intermediate-term sensitization. Although $25 \mathrm{~min}$ is a relatively short time interval, the absence of apparent sensitization 15 min after BST indicates that it takes some time to develop, unlike short-term sensitization, which becomes apparent within 2-5 min (Cohen et al. 1997; Antonov et al. 1999). Although intermediate-term sensitization lasts for a few hours, it is already present $25 \mathrm{~min}$ after training (Sutton et al. 2001). Moreover, intermediate-term facilitation of sensorimotor synapses in cell culture has been previously associated with the recruitment of pre-existing, but silent, synaptic structures (Kim et al. 2003). Prolonged contralateral training for LTS may, therefore, lead to an increase in the availability of immature structures, which, in turn, would require less training to formulate a sufficient number of additional synapses for the expression of intermediate-term sensitization.

Because short-term and intermediate-term sensitization can overlap in time, their mechanistic distinction is less ambiguous than the temporal one: Intermediate-term sensitization requires the synthesis of new proteins, but not of new mRNA, whereas short-term sensitization requires neither (Sutton et al. 2001). To clearly establish that the form of sensitization that becomes primed after 4-d contralateral LTS training is intermediate-term sensitization, it would be necessary to show that it can be blocked by protein synthesis inhibitors.

Although the same treatment of intact animals (i.e., 4-d contralateral LTS training) induces both the previously reported functionally silent restructuring of sensory neurons (Wainwright et al. 2002, 2004) and the priming of sensitization reported here, the two effects share a correlational, but not necessarily causal, relationship. A change in the potential for further sensitization could, in principle, be mediated by other, yet unidentified, effects of LTS training. For example, the 4-d training may increase the synthesis of enzymes that are necessary, and rate-limiting, for the forms of plasticity mediating sensitization. The lack of a priming effect of 4-d LTS training 15 min after BST suggests that 4-d training does not prime sensitization by merely enhancing the release of neuromodulators, or their effectiveness in inducing plasticity. Had the modulatory component of the network been potentiated by 4-d training, sensitization would have become apparent earlier than 25 min after BST (i.e., at the 15-min time point). This study thus, along with the recent report by Philips et al. (2006), constitutes an initial step in the cellular and molecular characterization of biological processes that can facilitate learning and memory.

\section{Acknowledgments}

We thank D. Fioravante, R. Mozzachiodi, and G. Phares for comments on an earlier version of the manuscript, and Jennifer DeLeon for her assistance with animal maintenance and testing. This work is supported by NIH grant NS 19895.

\section{References}

Antonov, I., Kandel, E.R., and Hawkins, R.D. 1999. The contribution of facilitation of monosynaptic PSPs to dishabituation and sensitization of the Aplysia siphon withdrawal reflex. J. Neurosci. 19: $10438-10450$.

Antzoulatos, E.G. and Byrne, J.H. 2004. Learning insights transmitted by glutamate. Trends Neurosci. 27: 555-560.

Bailey, C.H. and Chen, M. 1983. Morphological basis of long-term habituation and sensitization in Aplysia. Science 220: 91-93. . 1988a. Long-term sensitization in Aplysia increases the number of presynaptic contacts onto the identified gill motor neuron L7. Proc. Natl. Acad. Sci. 85: 9356-9359.

. 1988b. Long-term memory in Aplysia modulates the total number of varicosities of single identified sensory neurons. Proc. Natl. Acad. Sci. 85: 2373-2377.

. 1989. Time course of structural changes at identified sensory neuron synapses during long-term sensitization in Aplysia. J. Neurosci. 9: 1774-1780.

Carew, T.J., Castellucci, V.F., and Kandel, E.R. 1971. An analysis of dishabituation and sensitization of the gill-withdrawal reflex in Aplysia. Int. J. Neurosci. 2: 79-98.

Cohen, T.E., Kaplan, S.W., Kandel, E.R., and Hawkins, R.D. 1997. A simplified preparation for relating cellular events to behavior: Mechanisms contributing to habituation, dishabituation, and sensitization of the Aplysia gill-withdrawal reflex. J. Neurosci. 17: 2886-2899.

Kim, J.H., Udo, H., Li, H.L., Youn, T.Y., Chen, M., Kandel, E.R., and Bailey, C.H. 2003. Presynaptic activation of silent synapses and growth of new synapses contribute to intermediate and long-term facilitation in Aplysia. Neuron 40: 151-165.

Lamprecht, R. and LeDoux, J. 2004. Structural plasticity and memory. Nat. Rev. Neurosci. 5: 45-54.

Lubow, R.E. and Moore, A.U. 1959. Latent inhibition: The effect of nonreinforced pre-exposure to the conditional stimulus. J. Comp. Physiol. Psychol. 52: 415-419.

Philips, G.T., Tzvetkova, E.I., Marinesco, S., and Carew, T.J. 2006. Latent memory for sensitization in Aplysia. Learn. Mem. 13: 224-229.

\section{Learning \& Memory}


Pinsker, H.M., Hening, W.A., Carew, T.J., and Kandel, E.R. 1973. Long-term sensitization of a defensive withdrawal reflex in Aplysia. Science 182: 1039-1042.

Scholz, K.P. and Byrne, J.H. 1987. Long-term sensitization in Aplysia: Biophysical correlates in tail sensory neurons. Science 235: 685-687. Sutton, M.A., Masters, S.E., Bagnall, M.W., and Carew, T.J. 2001.

Molecular mechanisms underlying a unique intermediate phase of memory in Aplysia. Neuron 31: 143-154.

Tolman, E. 1949. There is more than one kind of learning. Psychol. Rev. 56: $144-154$
Wainwright, M.L., Zhang, H., Byrne, J.H., and Cleary, L.J. 2002. Localized neuronal outgrowth induced by long-term sensitization training in Aplysia. J. Neurosci. 22: 4132-4141.

Wainwright, M.L., Byrne, J.H., and Cleary, L.J. 2004. Dissociation of morphological and physiological changes associated with long-term memory in Aplysia. J. Neurophysiol. 92: 2628-2632.

Received February 27, 2006; accepted in revised form April 25, 2006. 


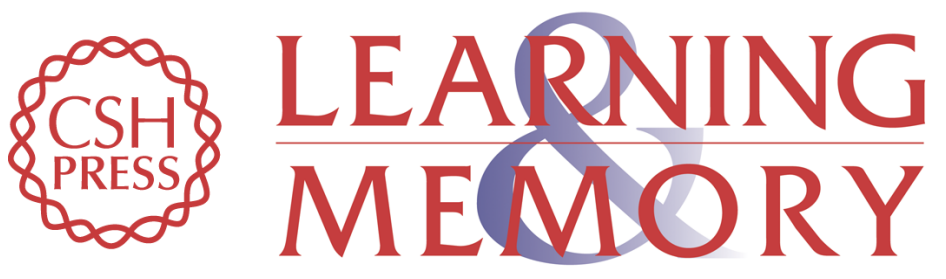

\section{Long-term sensitization training primes Aplysia for further learning}

Evangelos G. Antzoulatos, Marcy L. Wainwright, Leonard J. Cleary, et al.

Learn. Mem. 2006, 13:

Access the most recent version at doi:10.1101//m.230306

References This article cites 17 articles, 10 of which can be accessed free at: http://learnmem.cshlp.org/content/13/4/422.full.html\#ref-list-1

License

Email Alerting Receive free email alerts when new articles cite this article - sign up in the box at the Service top right corner of the article or click here. 